\title{
OPTIMAL STOPPING FOR PROCESSES WITH INDEPENDENT INCREMENTS, AND APPLICATIONS
}

\author{
G. DELIGIANNIDIS, * \\ H. LE ${ }^{* * * * *}$ AND \\ S. UTEV, ${ }^{* *}$ University of Nottingham
}

\begin{abstract}
In this paper we present an explicit solution to the infinite-horizon optimal stopping problem for processes with stationary independent increments, where reward functions admit a certain representation in terms of the process at a random time. It is shown that it is optimal to stop at the first time the process crosses a level defined as the root of an equation obtained from the representation of the reward function. We obtain an explicit formula for the value function in terms of the infimum and supremum of the process, by making use of the Wiener-Hopf factorization. The main results are applied to several problems considered in the literature, to give a unified approach, and to new optimization problems from the finance industry.
\end{abstract}

Keywords: Optimal stopping; random walk; Lévy process; option pricing

2000 Mathematics Subject Classification: Primary 62L15

Secondary 60G50

\section{Introduction}

Let $\left\{X_{t}\right\}_{t \in \mathcal{I}}$ be a process with stationary independent increments, where the time parameter is either discrete, $t \in \mathcal{I}=\mathbb{Z}^{+}=\{0,1,2, \ldots\}$, or continuous, $t \in \mathcal{I}=\mathbb{R}^{+}$.

For a given reward function $G$ and discount factor $\beta=\mathrm{e}^{-r}, r \geqslant 0$, we consider the problem of finding a pair $\left(V^{*}, \tau^{*}\right)$ such that $\tau^{*} \in \mathcal{T}$ and

$$
V^{*}(x)=V\left(x, \tau^{*}\right)=\mathrm{E}\left[\beta^{\tau^{*}} G^{+}\left(x+X_{\tau^{*}}\right)\right]=\sup _{\tau \in \mathcal{T}} \mathrm{E}\left[\beta^{\tau} G^{+}\left(x+X_{\tau}\right)\right],
$$

where $G^{+}(x)=\max \{G(x), 0\}$ and $\mathcal{T}$ is the set of all $\mathbb{F}$-stopping times. In the discrete case we define $\mathbb{F}=\left\{\mathcal{F}_{n}\right\}_{n \geq 0}$ to be the natural filtration of the random walk, while in the continuous case $\mathbb{F}=\left\{\mathcal{F}_{t}\right\}_{t \in \mathbb{R}^{+}}$is the augmented natural filtration of $\left\{X_{t}\right\}_{t \in \mathbb{R}^{+}}$that is right continuous and contains all P-null sets (see [15, p. 172]). We shall assume that the function $G$ and other functions considered in the paper are always Lebesgue measurable.

The cases with $G(x)=x^{+}, r=0$, and $G(x)=\left(\mathrm{e}^{x}-1\right)^{+}, r \geqslant 0$, in the discrete-time setting, were solved explicitly in [5]. The more general case with $G(x)=\left(K-\mathrm{e}^{x}\right)^{+}$and $G(x)=\left(\mathrm{e}^{x}-K\right)^{+}$was solved for random walks in [11]. By approximating a Lévy process by a sequence of random walks, the author also obtained the solution in continuous time. The

Received 7 April 2008; revision received 13 March 2009.

* Current address: Department of Mathematics, University of Leicester, University Road, Leicester, LE1 7RH, UK.

** Postal address: School of Mathematical Sciences, University of Nottingham, University Park, Nottingham, NG7 2RD, UK.

*** Email address: huiling.le@ nottingham.ac.uk 
solution for $G(x)=\left(x^{+}\right)^{v}, r=0$, in the discrete-time setting, was found in [13] for positive integer valued $v$. The same case was solved for continuous-time processes in [10]. Recently, the case for general $v>0$ and $r \geqslant 0$, for both continuous- and discrete-time parameters, was solved in [14].

In a slightly different direction in [3] and [4], the authors introduced a representation of the reward function in terms of a stream of payoffs. In the discrete case, they considered random walks whose increments admit a density. They obtained an explicit solution for the optimal stopping problem in the class of hitting times of semi-infinite intervals. Optimality in the class of all stopping times was obtained for monotone payoffs, under some regularity conditions on the density of the increments. In the continuous case, using analytical methods, they obtained a solution in the class of hitting times of semi-infinite intervals, with optimality in the class of all stopping times established for monotone payoffs.

In this paper we give an explicit solution to (1), for the general class of reward functions which admit the representation introduced in [3] and [4], and we prove that the optimal stopping time is indeed the hitting time of a semi-infinite interval. We present, in Section 2, the results for random walks and for Lévy processes. While so far the problems that have been solved in the literature involve monotone reward functions, we will demonstrate nonmonotone payoffs where our results can be applied successfully, obtaining optimality in the class of all stopping times. In Section 3 we apply our method to problems that have been solved in the literature, obtaining the same results, and also treat Canadian options in discrete time, a problem from the finance industry.

\section{Main results}

\subsection{Optimal stopping in discrete time}

Consider the random walk $\left\{X_{n}\right\}_{n \in \mathbb{Z}^{+}}$defined as the partial sums of an independent and identically distributed (i.i.d.) sequence of random variables, say $\xi, \xi_{1}, \xi_{2}, \ldots: X_{0}=0$ and $X_{n}=\sum_{k=1}^{n} \xi_{k}$ for $n>0$. Let $\mathbb{F}=\left\{\mathcal{F}_{n}\right\}_{n \geq 0}$ be the natural filtration of the random walk. We shall in the following assume that the discount factor satisfies $\beta=\mathrm{e}^{-r}<1$, unless otherwise stated.

The reward functions $G$ that we are interested in are those that have the representation

$$
G(x)=\sum_{n=0}^{\infty} \beta^{n} \mathrm{E}\left[g\left(x+X_{n}\right)\right]
$$

for some payoff function $g$.

Our result is closely linked with a geometric random time $T=T_{\beta}$, which is independent of the random walk $\left\{X_{n}\right\}_{n \in \mathbb{Z}^{+}}$and whose distribution is given by $\mathrm{P}(T \geqslant k)=\beta^{k}$ for $k \in \mathbb{Z}^{+}$. Note that, in terms of $T$, we can rewrite $G$ in (2) as

$$
G(x)=\frac{1}{1-\beta} \mathrm{E}\left[g\left(x+X_{T}\right)\right]
$$

Furthermore, let the random variables $I$ and $M$ be defined as follows:

$$
I \equiv I_{\beta}=\inf _{0 \leqslant n \leqslant T} X_{n} \quad \text { and } \quad M \equiv M_{\beta}=\sup _{0 \leqslant n \leqslant T} X_{n}
$$


It is shown in [7] and [9, pp. 177-178] that $X_{T}-M$ is independent of $M$ and equal in distribution to $I$. Thus, in particular,

$$
\mathrm{E}\left[\mathrm{e}^{X_{T}}\right]=\mathrm{E}\left[\mathrm{e}^{X_{T}-M}\right] \mathrm{E}\left[\mathrm{e}^{M}\right]=\mathrm{E}\left[\mathrm{e}^{\tilde{I}}\right] \mathrm{E}\left[\mathrm{e}^{M}\right],
$$

implying that

$$
X_{T} \stackrel{\mathrm{D}}{=} M+\tilde{I}
$$

where $\tilde{I}$ is a copy of $I$, independent of the random walk and of $T$. Note that by considering the reflected random walk $\left\{\hat{X}_{n}\right\}_{n \in \mathbb{Z}^{+}}=\left\{-X_{n}\right\}_{n \in \mathbb{Z}^{+}}$we also know that $X_{T}-I$ is independent of $I$ and equal in distribution to $M$.

The starting point of the proof of our main result is the following lemma obtained in [5].

Lemma 1. Let $\xi, \xi_{1}, \xi_{2}, \ldots$ be an i.i.d. sequence of real random variables, and define $X_{0}=0$, $X_{n}=\sum_{j=1}^{n} \xi_{j}$ for $n \geq 1$. Suppose that $h$ and $f$ are nonnegative functions, and that $\beta$ is $a$ constant satisfying $0 \leqslant \beta \leqslant 1$. If, for all $x$,

$$
f(x) \geqslant h(x) \text { and } f(x) \geqslant \mathrm{E}[\beta f(x+\xi)],
$$

then

$$
f(x) \geqslant \mathrm{E}\left[\beta^{\tau} h\left(x+X_{\tau}\right)\right]
$$

for all $x$ and $\mathbb{F}$-stopping times $\tau$.

The following is the first main result of the paper.

Theorem 1. Assume that the function $G(x)$ can be written in the form (2) for some function $g$.

(i) If there is an $x^{*}>-\infty$ such that

$$
\begin{gathered}
\mathrm{E}[g(x+M)] \text { is positive and nonincreasing for all } x<x^{*}, \\
\mathrm{E}[g(x+M)] \leqslant 0 \text { for all } x>x^{*},
\end{gathered}
$$

then the solution to (1) is given by the stopping time

$$
\tau^{*}=\inf \left\{n \geqslant 0: x+X_{n} \leqslant x^{*}\right\}
$$

and can be presented as

$$
V^{*}(x)=\frac{1}{1-\beta} \mathrm{E}\left[\mathbf{1}_{\left\{x+I \leqslant x^{*}\right\}} g\left(x+X_{T}\right)\right] .
$$

(ii) If there is an $x^{*}<\infty$ such that

$$
\begin{gathered}
\mathrm{E}[g(x+I)] \leqslant 0 \text { for all } x<x^{*}, \\
\mathrm{E}[g(x+I)] \text { is positive and nondecreasing for all } x>x^{*},
\end{gathered}
$$

then the solution to (1) is given by the stopping time $\tau^{*}=\inf \left\{n \geqslant 0: x+X_{n} \geqslant x^{*}\right\}$ and can be presented as

$$
V^{*}(x)=\frac{1}{1-\beta} \mathrm{E}\left[\mathbf{1}_{\left\{x+M \geqslant x^{*}\right\}} g\left(x+X_{T}\right)\right] .
$$


Proof. (i) Writing $W(x)=(1-\beta)^{-1} \mathrm{E}\left[\mathbf{1}_{\left\{x+I \leqslant x^{*}\right\}} g\left(x+X_{T}\right)\right]$, we first show that $W(x) \geqslant$ $V^{*}(x)$. For this, we proceed by showing that the functions $W(x)$ and $G^{+}(x)$ satisfy the conditions of Lemma 1.

By writing $\tilde{M}$ for a copy of $M$ which is independent of the random walk we have

$$
\begin{aligned}
W(x) & =\frac{1}{1-\beta} \mathrm{E}\left[\mathbf{1}_{\left\{x+I \leqslant x^{*}\right\}} g\left(x+\left(X_{T}-I\right)+I\right)\right] \\
& =\frac{1}{1-\beta} \mathrm{E}\left[\mathbf{1}_{\left\{x+I \leqslant x^{*}\right\}} g(x+\tilde{M}+I)\right] .
\end{aligned}
$$

However, it follows from the choice of $x^{*}$ that

$$
\mathrm{E}\left[\mathrm{E}\left[\mathbf{1}_{\left\{x+I>x^{*}\right\}} g(x+I+\tilde{M}) \mid I\right]\right] \leqslant 0,
$$

and so

$$
W(x) \geqslant \frac{1}{1-\beta} \mathrm{E}[g(x+I+\tilde{M})]=\frac{1}{1-\beta} \mathrm{E}\left[g\left(x+X_{T}\right)\right]=G(x) .
$$

The choice of $x^{*}$ also implies that $W(x) \geqslant 0$, and so what we have actually shown is that $W(x) \geqslant G^{+}(x)$.

To prove that $W(x)$ satisfies the second condition of Lemma 1, write

$$
F(x) \equiv \mathbf{1}_{\left(-\infty, x^{*}\right]}(x) \mathrm{E}[g(x+\tilde{M})] .
$$

Then

$$
W(x)=\frac{1}{1-\beta} \mathrm{E}[F(x+I)] .
$$

Let $J$ be an independent Bernoulli random variable with parameter $\beta$. Using characteristic functions, we can show (cf. [11]) that $I \stackrel{\mathrm{D}}{=}-J(\xi+I)^{-}$, where $x^{-}$is defined to be $\max (-x, 0)$. Thus,

$$
\begin{aligned}
\mathrm{E}[F(x+I)] & =\mathrm{E}\left[F\left(x-J(\xi+I)^{-}\right)\right] \\
& =(1-\beta) F(x)+\beta \mathrm{E}\left[F\left(x-(\xi+I)^{-}\right)\right] \\
& \geqslant \beta \mathrm{E}\left[F\left(x-(\xi+I)^{-}\right)\right] \\
& \geqslant \beta \mathrm{E}[F(x+\xi+I)],
\end{aligned}
$$

where the first inequality follows from the nonnegativity of $F$, and the second follows from the facts that $-x^{-} \leqslant x$ and that $F$ is nonincreasing, so that $W(x) \geqslant \mathrm{E}[\beta W(x+\xi)]$. It now follows from Lemma 1 that, for all stopping times $\tau$,

$$
W(x) \geqslant \mathrm{E}\left[\beta^{\tau} G^{+}\left(x+X_{\tau}\right)\right]
$$

so that $W(x) \geqslant V^{*}(x)$.

It remains to show that $W(x) \leqslant V^{*}(x)$ and that the stopping time $\tau^{*}$ defined in (5) is indeed optimal. For these results, it suffices to show that

$$
W(x) \leqslant \mathrm{E}\left[\beta^{\tau^{*}} G^{+}\left(x+X_{\tau^{*}}\right)\right] .
$$

Observe that $x+I \leqslant x^{*}$ if and only if $\tau^{*} \leqslant T$. Thus,

$$
W(x)=\frac{1}{1-\beta} \mathrm{E}\left[\mathbf{1}_{\left\{T \geqslant \tau^{*}\right\}} g\left(x+X_{\tau^{*}}+\left(I-X_{\tau^{*}}\right)+\tilde{M}\right)\right] .
$$


By the memoryless property of the geometric distribution and the strong Markov property of $\left\{X_{n}\right\}_{n \in \mathbb{Z}^{+}}$, on the event $\left\{T \geqslant \tau^{*}\right\}, I-X_{\tau^{*}}$ is independent of $\mathcal{F}_{\tau^{*}}$ and is equal in distribution to $I$. Thus, we replace $I-X_{\tau^{*}}$ by a copy of $I$, denoted by $\tilde{I}$, which is independent of both the random walk $\left\{X_{n}\right\}_{n \in \mathbb{Z}^{+}}$and $\tilde{M}$. This gives

$$
\begin{aligned}
W(x) & =\frac{1}{1-\beta} \mathrm{E}\left[\mathbf{1}_{\left\{T \geqslant \tau^{*}\right\}} g\left(x+X_{\tau^{*}}+\tilde{I}+\tilde{M}\right)\right] \\
& =\frac{1}{1-\beta} \mathrm{E}\left[\mathrm{E}\left[\mathbf{1}_{\left\{T \geqslant \tau^{*}\right\}} g\left(x+X_{\tau^{*}}+\tilde{X}_{\tilde{T}}\right) \mid \mathcal{F}_{\tau^{*}}\right]\right] \\
& =\mathrm{E}\left[\beta^{\tau^{*}} \mathrm{E}\left[G\left(x+X_{\tau^{*}}\right) \mid \mathcal{F}_{\tau^{*}}\right]\right] \\
& =\mathrm{E}\left[\beta^{\tau^{*}} G\left(x+X_{\tau^{*}}\right)\right] \\
& \leqslant \mathrm{E}\left[\beta^{\tau^{*}} G^{+}\left(x+X_{\tau^{*}}\right)\right]
\end{aligned}
$$

where in the second equality $\tilde{X}_{\tilde{T}}$ is an independent copy of the random walk evaluated at an independent copy of the geometric time $\tilde{T}$.

(ii) This can be reduced to case (i) by the transformation $\left(x, X_{n}\right) \mapsto\left(-x,-X_{n}\right)$.

Remark 1. The last inequality in the above proof is actually an equality. To see this, we only need to show that $G\left(x+X_{\tau^{*}}\right) \geqslant 0$. It follows from the definition of $\tau^{*}$ that $x+X_{\tau^{*}} \leqslant x^{*}$. Thus, for $\tilde{I} \stackrel{\mathrm{D}}{=} I$ and $\tilde{M} \stackrel{\mathrm{D}}{=} M$, independent of each other and both also independent of $\left\{X_{t}\right\}_{t \in \mathbb{R}^{+}}$, we have $x+X_{\tau^{*}}+\tilde{I} \leqslant x^{*}$, and so $\mathrm{E}\left[g\left(x+X_{\tau^{*}}+\tilde{I}+\tilde{M}\right) \mid X_{\tau^{*}}, \tilde{I}\right] \geqslant 0$, implying that $G\left(x+X_{\tau^{*}}\right) \geqslant 0$.

Remark 2. (a) The results of Theorem 1 hold even when $W(x)$ is infinite. In that case, our proof establishes that the solution $V^{*}(x)$ of the optimal stopping problem must also be infinite.

(b) It is clear from the proof that the solution of (1) is the same as the solution of (1) with $G^{+}(x)$ replaced by $G(x)$.

(c) If $g$ is decreasing then the monotonicity assumption on $\mathrm{E}[g(x+M)]$ in Theorem 1(i) is satisfied. Similarly, if $g$ is increasing then the monotonicity assumption on $\mathrm{E}[g(x+I)]$ in Theorem 1(ii) is satisfied.

\subsection{Optimal stopping in continuous time}

In the continuous-time setting, consider a Lévy process $\left\{X_{t}\right\}_{t \in \mathbb{R}^{+}}$starting from the origin. We assume that the sample paths of $\left\{X_{t}\right\}_{t \in \mathbb{R}^{+}}$are almost surely right continuous with left limits and let $\mathbb{F}=\left\{\mathcal{F}_{t}\right\}_{t \in \mathbb{R}^{+}}$be its augmented natural filtration. The discount factor is given by $\beta=\mathrm{e}^{-r}$ with $r>0$. We also introduce an independent exponential random variable $T=T_{r}$ with parameter $r$ and define

$$
I \equiv I_{r}=\inf _{0 \leqslant t \leqslant T} X_{t} \quad \text { and } \quad M \equiv M_{r}=\sup _{0 \leqslant t \leqslant T} X_{t} .
$$

Then, the results on the Wiener-Hopf factorization of Lévy processes given in [8] and [9, Theorem 6.16] show that

$$
X_{T}-M \text { is independent of } M \quad \text { and } \quad X_{T}-M \stackrel{\mathrm{D}}{=} I .
$$

Using similar arguments to those given in the proof of Lemma 1 (see [5]), we obtain a continuous-time analogue which we state below. For a different version of this result, see [9, Lemma 9.1]. 
Lemma 2. Let $\left\{X_{t}\right\}_{t \in \mathbb{R}^{+}}$be a Lévy process with $X_{0}=0$. Suppose further that $f$ and $h$ are measurable, nonnegative functions, and that $r>0$. If, for all $x, f(x) \geq h(x)$, and $\left\{\mathrm{e}^{-r t} f\left(x+X_{t}\right)\right\}_{t \in \mathbb{R}^{+}}$is a right-continuous supermartingale, then

$$
f(x) \geq \mathrm{E}\left[\mathrm{e}^{-r \tau} h\left(x+X_{\tau}\right)\right]
$$

for all $x$ and $\mathbb{F}$-stopping times $\tau$.

We have the following result on the optimal stopping problem for Lévy processes, where we assume that the reward function $G$ has the representation

$$
G(x)=\int_{0}^{\infty} \mathrm{e}^{-r t} \mathrm{E}\left[g\left(x+X_{t}\right)\right] \mathrm{d} t
$$

for some payoff function $g$.

Theorem 2. Assume that the function $G(x)$ has the representation (7), where $g$ is continuous.

(i) If there is an $x^{*}>-\infty$ such that

$$
\begin{gathered}
\mathrm{E}[g(x+M)] \text { is positive and nonincreasing for all } x<x^{*}, \\
\mathrm{E}[g(x+M)] \leqslant 0 \text { for all } x>x^{*},
\end{gathered}
$$

then the solution of $(1)$ is given by the optimal stopping time $\tau^{*}=\inf \left\{t \geqslant 0: x+X_{t} \leqslant x^{*}\right\}$ and can be written as

$$
V^{*}(x)=r^{-1} \mathrm{E}\left[\mathbf{1}_{\left\{x+I \leqslant x^{*}\right\}} g\left(x+X_{T}\right)\right] .
$$

(ii) If there is an $x^{*}<\infty$ such that

$$
\begin{gathered}
\mathrm{E}[g(x+I)] \leqslant 0 \text { for all } x<x^{*}, \\
\mathrm{E}[g(x+I)] \text { is positive and nondecreasing for all } x>x^{*},
\end{gathered}
$$

then the solution of (1) is given by the optimal stopping time $\tau^{*}=\inf \left\{t \geqslant 0: x+X_{t} \geqslant x^{*}\right\}$ and can be written as

$$
V^{*}(x)=r^{-1} \mathrm{E}\left[\mathbf{1}_{\left\{x+M \geqslant x^{*}\right\}} g\left(x+X_{T}\right)\right] .
$$

Proof. The proof is similar to that for the discrete case and so, in the following, we only outline the proof for case (i).

Writing $W(x)=r^{-1} \mathrm{E}\left[\mathbf{1}_{\left\{x+I \leqslant x^{*}\right\}} g\left(x+X_{T}\right)\right]$, we show first that $W(x) \geqslant G^{+}(x)$. Using the result on the Wiener-Hopf factorization of Lévy processes, we can write

$$
W(x)=r^{-1} \mathrm{E}\left[\mathbf{1}_{\left\{x+I \leqslant x^{*}\right\}} g(x+\tilde{M}+I)\right],
$$

where $\tilde{M}$ is a copy of $M$, independent of $X_{t}$. By our assumptions, on the event $\left\{x+I>x^{*}\right\}$, we have

$$
\mathrm{E}\left[\mathbf{1}_{\left\{x+I>x^{*}\right\}} g(x+I+\tilde{M})\right] \leqslant 0,
$$

and, thus,

$$
W(x) \geqslant r^{-1} \mathrm{E}[g(x+I+\tilde{M})]=r^{-1} \mathrm{E}\left[g\left(x+X_{T}\right)\right]=G(x),
$$

so that $W(x) \geqslant G^{+}(x)$ as $W(x) \geqslant 0$. 
Next, we show that $\left\{\mathrm{e}^{-r t} W\left(x+X_{t}\right)\right\}_{t \in \mathbb{R}^{+}}$is a right-continuous supermartingale. For this, we write $W(x)=r^{-1} \mathrm{E}[F(x+I)]$, where $F(x)=\mathbf{1}_{\left(-\infty, x^{*}\right]}(x) \mathrm{E}[g(x+M)]$. If $\tilde{I}$ is a copy of $I$, independent of the Lévy process and of $T$, then, on the event $\{T>t\}, I$ is equal in distribution to $\left(X_{t}+\tilde{I}\right) \wedge I_{t}$, where $I_{t}=\min _{0 \leqslant s \leqslant t} X_{s}$ (cf. [9, p. 243]). Since $F(x)$ is nonnegative and nonincreasing,

$$
\begin{aligned}
W(x) & \geqslant r^{-1} \mathrm{E}\left[\mathbf{1}_{\{T>t\}} F(x+I)\right] \\
& =r^{-1} \mathrm{E}\left[\mathbf{1}_{\{T>t\}} F\left(x+\min \left\{\left(X_{t}+\tilde{I}\right), I_{t}\right\}\right)\right] \\
& \geqslant r^{-1} \mathrm{E}\left[\mathbf{1}_{\{T>t\}} F\left(x+X_{t}+\tilde{I}\right)\right] \\
& =r^{-1} \mathrm{E}\left[\mathrm{e}^{-r t} F\left(x+X_{t}+\tilde{I}\right)\right] \\
& =\mathrm{E}\left[\mathrm{e}^{-r t} W\left(x+X_{t}\right)\right] .
\end{aligned}
$$

Thus, the fact that $\left\{\mathrm{e}^{-r t} W\left(X_{t}\right)\right\}_{t \in \mathbb{R}^{+}}$is a right-continuous supermartingale follows from the continuity of $g$. Then, by Lemma 2 , since, for all $x, W(x) \geq G^{+}(x) \geq 0$ and $\left\{\mathrm{e}^{-r t} W\left(X_{t}\right)\right\}_{t \in \mathbb{R}^{+}}$ is a nonnegative supermartingale,

$$
\left.W(x) \geqslant \mathrm{E}\left[\mathrm{e}^{-r \tau} W\left(x+X_{\tau}\right)\right] \geqslant \mathrm{E}^{-r \tau} \mathrm{e}^{+}\left(x+X_{\tau}\right)\right]
$$

for all $\tau$ under the given assumptions. The arbitrary nature of $\tau$ implies that $W(x) \geqslant V^{*}(x)$, noting that $\beta=\mathrm{e}^{-r}$.

On the other hand, $x+I \leqslant x^{*}$ if and only if $T \geqslant \tau^{*}$. Also, on the event $\left\{T \geqslant \tau^{*}\right\}, X_{T}-X_{\tau^{*}}$ is independent of $\mathcal{F}_{\tau^{*}}$, by the strong Markov property, and it is equal in distribution to $X_{T}$, by the memoryless property of the exponential distribution. Then, if $\left\{\tilde{X}_{t}\right\}_{t \in \mathbb{R}^{+}}$is an independent copy of the Lévy process, and $\tilde{T}$ is a copy of $T$, independent of $X_{t}, \tilde{X}_{t}$, and $T$,

$$
\begin{aligned}
W(x) & =r^{-1} \mathrm{E}\left[\mathbf{1}_{\left\{T \geqslant \tau^{*}\right\}} g\left(x+\left(X_{T}-X_{\tau^{*}}\right)+X_{\tau^{*}}\right)\right] \\
& =r^{-1} \mathrm{E}\left[\mathbf{1}_{\left\{T \geqslant \tau^{*}\right\}} g\left(x+\tilde{X}_{\tilde{T}}+X_{\tau^{*}}\right)\right] \\
& =\mathrm{E}\left[\mathrm{e}^{-r \tau^{*}} \mathrm{E}\left[\int_{0}^{\infty} \mathrm{e}^{-r t} g\left(x+X_{\tau^{*}}+\tilde{X}_{t}\right) \mathrm{d} t \mid \tilde{F}_{\tau^{*}}\right]\right] \\
& =\mathrm{E}\left[\mathrm{e}^{-r \tau^{*}} G\left(x+X_{\tau^{*}}\right)\right] \\
& \leqslant \mathrm{E}\left[\mathrm{e}^{-r \tau^{*}} G^{+}\left(x+X_{\tau^{*}}\right)\right] .
\end{aligned}
$$

Hence, $\tau^{*}$ is indeed the optimal stopping time and $W(x)$ is the value function of the continuoustime optimal stopping problem (1).

\section{Examples and applications}

In this section we demonstrate how the results described in Section 2 can be applied to a wide class of functions, recalling that $T, I$, and $M$ are those defined at the beginning of Section 2.1 in the discrete-time setting, and those defined at the beginning of Section 2.2 in the continuous-time setting.

\subsection{Linear reward functions}

First, we treat the linear reward function $G(x)=x$ and some perturbations to demonstrate how Theorems 1 and 2 work and when the conditions of our results are satisfied. We start with the discrete-time setting. 
(a) Let $G(x)=x$, and recall that $\beta<1$. Note that the case in which $\beta=1$ has already been treated in [5]. Assume for now that $\mathrm{E}[\xi]=\mu$. It is easy to see that if we take $g(x)=$ $(1-\beta) x-\beta \mu$ then $G(x)=\sum_{n \geqslant 0} \beta^{n} \mathrm{E}\left[g\left(x+X_{n}\right)\right]$. Clearly, $g(x)$ is an increasing function of $x$. To find the optimal barrier, we solve the following equation for $x^{*}$ :

$$
(1-\beta) x^{*}+(1-\beta) \mathrm{E}[I]-\mu \beta=0 .
$$

Since $\mathrm{E}\left[X_{T_{\beta}}\right]=\mu \beta /(1-\beta)$ and $\mathrm{E}\left[X_{T_{\beta}}\right]=\mathrm{E}[I]+\mathrm{E}[M]$, we have

$$
\mathrm{E}[I]=\frac{\mu \beta}{1-\beta}-\mathrm{E}[M] .
$$

Expressing $\mathrm{E}[I]$ in (10) in terms of $\mathrm{E}[M]$ shows that $x^{*}=\mathrm{E}[M]$. By Theorem 1(ii), the optimal stopping time is then given by

$$
\tau^{*}=\inf \left\{n \geqslant 0: x+X_{n} \geqslant \mathrm{E}[M]\right\} .
$$

Denoting the indicator function $\mathbf{1}_{\{x+M \geqslant \mathrm{E}[M]\}}$ by $\boldsymbol{I}$ to simplify notation, the solution is given by

$$
\begin{aligned}
(1-\beta) V^{*}(x) & =\mathrm{E}[\boldsymbol{I}((1-\beta)(x+M+I)-\mu \beta)] \\
& =\mathrm{E}\left[\boldsymbol{I}((1-\beta)(x+M)-\mu \beta)+\boldsymbol{I}(1-\beta)\left(\frac{\mu \beta}{1-\beta}-\mathrm{E}[M]\right)\right],
\end{aligned}
$$

since $I$ is independent of $M$ and hence of $\boldsymbol{I}$. This gives

$$
(1-\beta) V^{*}(x)=\mathrm{E}[\boldsymbol{I}(1-\beta)(x+M-\mathrm{E}[M])],
$$

so that

$$
V^{*}(x)=\mathrm{E}[\boldsymbol{I}(x+M-\mathrm{E}[M])]=\mathrm{E}\left[(x+M-\mathrm{E}[M])^{+}\right] .
$$

In particular, by letting $\beta \uparrow 1$ we recover the solution obtained in [5] for the same reward function but with $\beta=1$.

(b) We now consider a nondegenerate symmetric integer-valued random walk and the perturbed reward function $G(x)=x+(-1)^{x} c$, where $x \in \mathbb{Z}$ and $c$ is a constant. Direct calculation shows that $G(x)$ has the representation (2) with $g(x)=(1-\beta)\left\{x+(-1)^{x} c \delta\right\}$, where $\delta=$ $(1-q \beta) /(1-\beta)>1$ and $q=\mathrm{E}\left[(-1)^{\xi}\right]$. We have $\mathrm{E}\left[(-1)^{I}\right] \mathrm{E}\left[(-1)^{M}\right]=\mathrm{E}\left[(-1)^{X_{T}}\right]$, by Wiener-Hopf factorization, and that $\mathrm{E}\left[(-1)^{I}\right]=\mathrm{E}\left[(-1)^{M}\right]$, by symmetry. Hence,

$$
\mathrm{E}[g(x+I)]=(1-\beta)\left\{x+\mathrm{E}[I]+(-1)^{x} c \sqrt{\delta}\right\},
$$

which is nondecreasing provided that $1 \geqslant 2|c \sqrt{\delta}|$. This condition for $\mathrm{E}[g(x+I)]$ to be nondecreasing is clearly weaker than requiring that $g$ is nondecreasing, i.e. $1 \geqslant 2|c \delta|$.

(c) By restricting to the simple symmetric random walk, we are able to consider a much broader class of functions. Let $\left\{X_{n}\right\}_{n \in \mathbb{Z}^{+}}$be a simple symmetric random walk so that the i.i.d. increments $\xi$ are such that $\xi=1$ with probability $\frac{1}{2}$ and $\xi=-1$ with probability $\frac{1}{2}$. Let $M_{n}=\max _{0 \leqslant i \leqslant n} X_{i}$ be the maximum of the random walk up to time $n$, and let $b \in \mathbb{Z}^{+}$. Then, for $k>0$, by the reflection principle, it is true that

$$
\mathrm{P}\left(M_{n}=k\right)=\mathrm{P}\left(X_{n}=k\right)+\mathrm{P}\left(X_{n}=k+1\right),
$$


which obviously also holds for $k=0$ by symmetry. Hence,

$$
\mathrm{P}(M=k)=\mathrm{P}\left(X_{T}=k\right)+\mathrm{P}\left(X_{T}=k+1\right), \quad k \in \mathbb{Z}^{+},
$$

and so

$$
\mathrm{E}[g(x+M)]=\sum_{k \geqslant 1}\{g(x+k)+g(x+k-1)\} \mathrm{P}\left(X_{T}=k\right)+g(x) \mathrm{P}\left(X_{T}=0\right) .
$$

For $\mathrm{E}[g(x+M)]$ to be a nonincreasing function of $x \in \mathbb{Z}$, it is sufficient to require $g(x)+g(x+1)$ to be nonincreasing for integer valued $x$, and $g(x)$ nonincreasing for $x<x^{*}$, which is clearly weaker than the assumption that $g$ is globally decreasing.

As for the discrete-time setting, Theorem 2 does not hold just for monotone functions, as the following demonstrates.

(d) Assume that $\left\{X_{t}\right\}_{t \in \mathbb{R}^{+}}$is a Lévy process starting from the origin such that the negative of its infimum at the exponential time $T$ has exponential distribution with parameter $\lambda$. Note that Brownian motion and spectrally positive Lévy processes have this property. For simplicity, we assume that $\lambda=1$. Consider the perturbed reward function $G(x)=x+c_{1}+c_{2} \cos (x)+$ $c_{3} \sin (x)$. The function $G(x)$ has the representation (2) with $g(x)=x+\tilde{c}_{1}+\tilde{c}_{2} \cos (x)+$ $\tilde{c}_{3} \sin (x)$, where the relations between the coefficients can be easily obtained. A particular case is $g(x)=x+1+\sin (x)+\cos (x)$, which is clearly not monotone. However, $\mathrm{E} g(x+I)=x+\sin (x)$ is monotone.

This can be extended to a broader class of functions. Observe that

$$
\mathrm{E}[g(x+I)]=\int_{0}^{\infty} g(x-y) \mathrm{e}^{-y} \mathrm{~d} y,
$$

and so, if $g$ is differentiable,

$$
\frac{\mathrm{d}}{\mathrm{d} x} \mathrm{E}[g(x+I)]=\int_{-\infty}^{x} g^{\prime}(z) \mathrm{e}^{z-x} \mathrm{~d} z .
$$

Thus, if $g$ has the property that $\int_{-\infty}^{x} g^{\prime}(z) \mathrm{e}^{z} \mathrm{~d} z>0$ almost everywhere then, if $\mathrm{E}[g(x+I)]=0$ has a root $x^{*}$, it must be unique. Also, $\mathrm{E}[g(x+I)]$ is nondecreasing in $x$, so that Theorem 2(ii) can be applied to $G$.

\subsection{Exponential reward functions}

Here we consider exponential reward functions in the discrete-time setting. In particular, we apply Theorem 1 to perpetual American call and put options and, similarly to the previous subsection, also consider perturbations. We write $a=\mathrm{E}\left[\mathrm{e}^{\xi}\right]$, and assume that $a \beta<1$.

(a) Perpetual American call. The price of a perpetual American call option with strike $K$, under the random walk model, is the solution of the optimal stopping problem

$$
V^{*}(x)=\sup _{\tau \in \mathcal{T}} \mathrm{E}\left[\beta^{\tau}\left(\mathrm{e}^{x+X_{\tau}}-K\right)^{+}\right],
$$

so that it corresponds to the case $G(x)=\mathrm{e}^{x}-K$. Note that the solution for this problem with $K=1$ was obtained in [5] using a different method.

Observe that, if $g(x)=(1-a \beta) \mathrm{e}^{x}-(1-\beta) K$ then

$$
\sum_{n=0}^{\infty} \beta^{n} \mathrm{E}\left[g\left(x+X_{n}\right)\right]=\mathrm{e}^{x}-K .
$$


Since $g$ is an increasing function and

$$
\mathrm{E}\left[\mathrm{e}^{X_{T}}\right]=\frac{1-\beta}{1-a \beta},
$$

we apply Theorem 1(ii) to deduce that the optimal stopping barrier is the solution $x^{*}$ of

$$
\mathrm{E}\left[(1-a \beta) \mathrm{e}^{x^{*}+I}-(1-\beta) K\right]=0 .
$$

Hence,

$$
\mathrm{e}^{x^{*}}=\frac{1-\beta}{1-a \beta} \frac{K}{\mathrm{E}\left[\mathrm{e}^{I}\right]}, \quad \text { i.e. } \mathrm{e}^{x^{*}}=K \mathrm{E}\left[\mathrm{e}^{M}\right]
$$

by (3) and (11), and so the optimal stopping time is

$$
\tau^{*}=\inf \left\{n \geqslant 0: x+X_{n} \geqslant \ln \left(K \mathrm{E}\left[\mathrm{e}^{M}\right]\right)\right\}
$$

The value function is

$$
V^{*}(x)=\frac{1}{1-\beta} \mathrm{E}\left[\mathbf{1}_{\left\{x+M \geqslant x^{*}\right\}}\left((1-a \beta) \mathrm{e}^{x+X_{T}}-(1-\beta) K\right)\right],
$$

and so, writing $\boldsymbol{I}$ for the indicator function $\mathbf{1}_{\left\{x+M \geqslant x^{*}\right\}}$, we obtain

$$
\begin{aligned}
V^{*}(x) & =\mathrm{E}\left[\mathrm{E}\left[\boldsymbol{I}\left(\frac{1-a \beta}{1-\beta} \mathrm{e}^{x+I+M}-K\right) \mid M\right]\right] \\
& =\mathrm{E}\left[\boldsymbol{I}\left(\frac{\mathrm{e}^{x+M}}{\mathrm{E}\left[\mathrm{e}^{M}\right]}-K\right)\right] \\
& =\frac{\mathrm{E}\left[\left(\mathrm{e}^{x+M}-K \mathrm{E}\left[\mathrm{e}^{M}\right]\right)^{+}\right]}{\mathrm{E}\left[\mathrm{e}^{M}\right]},
\end{aligned}
$$

agreeing with the solution given in [11].

(b) Perpetual American put. Similarly, for a perpetual American put, the reward function can be expressed in terms of the function $g=K(1-\beta)-(1-a \beta) \mathrm{e}^{x}$ as

$$
K-\mathrm{e}^{x}=\sum_{n \geqslant 0} \beta^{n} \mathrm{E}\left[g\left(x+X_{n}\right)\right] .
$$

The function $g$ is decreasing and, thus, Theorem 1(i) gives the optimal stopping time as

$$
\tau^{*}=\inf \left\{n \geqslant 0: x+X_{n} \leqslant \ln \left(K \mathrm{E}\left[\mathrm{e}^{I}\right]\right)\right\}
$$

and the price as

$$
V^{*}(x)=\frac{\mathrm{E}\left[\left(K \mathrm{E}\left[\mathrm{e}^{I}\right]-\mathrm{e}^{x+I}\right)^{+}\right]}{\mathrm{E}\left[\mathrm{e}^{I}\right]},
$$

again agreeing with the solution given in [11]. 
(c) Let $\left\{X_{n}\right\}_{n \in \mathbb{Z}^{+}}$be a random walk such that the distribution of the negative of its infimum at an independent geometric time $T$ is a mixture of an atom at zero and an exponential distribution with parameter 1 . To see that such a random walk exists, consider the descending ladder process of the random walk. The number of descending ladder points, up to an independent geometric time, is also geometric (see [7]), say of parameter $p$, and is independent of the ladder height process. Thus, the distribution of the negative of the infimum is the geometric compounding of the distribution of the descending ladder heights. If the descending ladder heights are exponentially distributed then, conditional on the geometric time not being 0 , the infimum of the random walk will also have exponential distribution. An example of a random walk with exponentially distributed descending ladder heights is given in [6, p. 193], where the increments are distributed as the difference of two independent exponential random variables.

We note that, as in example (d) of Section 3.1, if $G(x)=K \mathrm{e}^{x}+c_{1}+c_{2} \cos (x)+c_{3} \sin (x)$ then $G(x)$ has the representation (2) with $g(x)=K \mathrm{e}^{x}+\tilde{c}_{1}+\tilde{c}_{2} \cos (x)+\tilde{c}_{3} \sin (x)$. For example, if

$$
\begin{aligned}
G(x)= & \frac{1-\beta}{1-a \beta} \mathrm{e}^{x}-2+\left\{\mathrm{E}\left[\sin \left(X_{T}\right)\right]-\mathrm{E}\left[\cos \left(X_{T}\right)\right]\right\} \cos (x) \\
& +\left\{\mathrm{E}\left[\cos \left(X_{T}\right)\right]+\mathrm{E}\left[\sin \left(X_{T}\right)\right]\right\} \sin (x),
\end{aligned}
$$

then $g(x)=\mathrm{e}^{x}-2+\sin (x)-\cos (x)$, which is nonmonotone. For such a $g$,

$$
\mathrm{E}[g(x+I)]=(1-p) g(x)+p\left\{\frac{\mathrm{e}^{x}}{2}-2-\cos (x)\right\} .
$$

It can be checked that, for all $p>0.4, \mathrm{E}[g(x+I)]=0$ has a unique solution $x^{*}$. The derivative of $F(x)=\mathbf{1}_{\left[x^{*}, \infty\right)}(x) \mathrm{E}[g(x+I)]$ is given by

$$
F^{\prime}(x)=(1-p)\left\{\mathrm{e}^{x}-\sin (x)+\cos (x)\right\}+p\left\{\frac{\mathrm{e}^{x}}{2}+\sin (x)\right\}
$$

for $x>x^{*}$, which again can be checked to be nonnegative for $x>x^{*}$ when $p>0.4$. Thus, $F(x)$ is nondecreasing and Theorem 1(ii) can be applied to $G$.

\subsection{Canadian options}

Canadian options have two rewards, a boundary payoff and a final payoff. The option, with strike $K$, can be exercised at any time before maturity to receive the boundary payoff $\left(K-\mathrm{e}^{x}\right)^{+}$, or at maturity to receive the final payoff $f(x) \geqslant 0$. Under the random walk model, the maturity $\tilde{T}$ of the Canadian option is a random variable which has a geometric distribution and is independent of the random walk. We continue to write $a=\mathrm{E}\left[\mathrm{e}^{\xi}\right]$ and assume that the parameter for the distribution of $\tilde{T}$ is $\alpha$. We further assume that the following condition on the derivative of $f$ holds:

$$
f^{\prime}(x) \geqslant-\frac{1-a \gamma}{1-\alpha} \mathrm{e}^{x},
$$

where $\gamma=\alpha \beta$, and assume that $a \gamma<1$.

The price for the Canadian option is then given by

$$
\tilde{V}^{*}(x)=\sup _{\tau \in \mathcal{T}} \mathrm{E}\left[\beta^{\tau}\left(K-\mathrm{e}^{x+X_{\tau}}\right)^{+} \mathbf{1}_{\{\tau \leqslant \tilde{T}\}}+\beta^{\tilde{T}} f\left(x+X_{\tilde{T}}\right) \mathbf{1}_{\{\tau>\tilde{T}\}}\right] .
$$


Observe that

$$
\begin{aligned}
\tilde{V}^{*}(x)= & \sup _{\tau \in \mathcal{T}} \mathrm{E}\left[\beta^{\tau}\left(K-\mathrm{e}^{x+X_{\tau}}\right)^{+} \mathbf{1}_{\{\tau \leqslant \tilde{T}\}}+\beta^{\tilde{T}} f\left(x+X_{\tilde{T}}\right) \mathbf{1}_{\{\tau>\tilde{T}\}}\right] \\
= & \mathrm{E}\left[\beta^{\tilde{T}} f\left(x+X_{\tilde{T}}\right)\right] \\
& +\sup _{\tau \in \mathcal{T}} \mathrm{E}\left[\left\{\gamma^{\tau}\left(K-\mathrm{e}^{x+X_{\tau}}\right)^{+}-\beta^{\tilde{T}} f\left(x+X_{\tilde{T}}\right)\right\} \mathbf{1}_{\{\tau \leqslant \tilde{T}\}}\right] .
\end{aligned}
$$

Thus, solving (12) is equivalent to solving the optimal stopping problem

$$
\bar{V}^{*}(x)=\sup _{\tau \in \mathcal{T}} \mathrm{E}\left[\left\{\gamma^{\tau}\left(K-\mathrm{e}^{x+X_{\tau}}\right)^{+}-\beta^{\tilde{T}} f\left(x+X_{\tilde{T}}\right)\right\} \mathbf{1}_{\{\tau \leqslant \tilde{T}\}}\right] .
$$

For this, we first re-express the second term in the above equation as follows:

$$
\begin{aligned}
\mathrm{E}\left[\beta^{\tilde{T}} f\left(x+X_{\tilde{T}}\right) \mathbf{1}_{\{\tau \leqslant \tilde{T}\}}\right] & =\mathrm{E}\left[\beta^{\tau} \sum_{n=0}^{\infty} \mathrm{E}\left[\beta^{n} f\left(x+X_{\tau}+\tilde{X}_{n}\right) \mid \mathcal{F}_{\tau}\right] \mathrm{E}\left[\mathbf{1}_{\{\tilde{T}=\tau+n\}} \mid \mathcal{F}_{\tau}\right]\right] \\
& =\mathrm{E}\left[\gamma^{\tau} \sum_{n=0}^{\infty} \mathrm{E}\left[(1-\alpha) \gamma^{n} f\left(x+X_{\tau}+\tilde{X}_{n}\right) \mid \mathcal{F}_{\tau}\right]\right] \\
& =\frac{1-\alpha}{1-\gamma} \mathrm{E}\left[\gamma^{\tau} \mathrm{E}\left[f\left(x+X_{\tau}+\tilde{X}_{\bar{T}}\right) \mid \mathcal{F}_{\tau}\right]\right],
\end{aligned}
$$

where $\left\{\tilde{X}_{n}\right\}_{n \in \mathbb{Z}^{+}}$is an i.i.d. copy of $\left\{X_{n}\right\}_{n \in \mathbb{Z}^{+}}$, and $\bar{T}$ is a geometric random variable which has parameter $\gamma$ and is independent of the random walk. Write

$$
H(x)=\frac{1-\alpha}{1-\gamma} \mathrm{E}\left[f\left(x+X_{\bar{T}}\right)\right]
$$

Then, (13) becomes

$$
\tilde{V}^{*}(x)=\sup _{\tau \in \mathcal{T}} \mathrm{E}\left[\gamma^{\tau}\left\{\left(K-\mathrm{e}^{x+X_{\tau}}\right)^{+}-H\left(x+X_{\tau}\right)\right\}\right] .
$$

Since $H$ is nonnegative, $\left(\left(K-\mathrm{e}^{x}\right)^{+}-H(x)\right)^{+}=\left(K-\mathrm{e}^{x}-H(x)\right)^{+}$. However, as noted in Remark 2 after the proof of Theorem 1, the solution for (14) is identical to the solution for the optimal stopping problem,

$$
\begin{aligned}
V^{*}(x) & =\sup _{\tau \in \mathcal{T}} \mathrm{E}\left[\gamma^{\tau}\left(\left(K-\mathrm{e}^{x+X_{\tau}}\right)^{+}-H\left(x+X_{\tau}\right)\right)^{+}\right] \\
& =\sup _{\tau \in \mathcal{T}} \mathrm{E}\left[\gamma^{\tau}\left(K-\mathrm{e}^{x+X_{\tau}}-H\left(x+X_{\tau}\right)\right)^{+}\right] .
\end{aligned}
$$

Hence, if we write $g(x)=K(1-\gamma)-(1-a \gamma) \mathrm{e}^{x}-(1-\alpha) f(x)$, we have

$$
K-\mathrm{e}^{x}-H(x)=\sum_{n=0}^{\infty} \gamma^{n} \mathrm{E}\left[g\left(x+X_{n}\right)\right] .
$$

Assume that there is an $x^{*} \in \mathbb{R}$ such that $g(x)$ satisfies the assumptions of Theorem 1(i). Then, it follows from Theorem 1 that, if $I_{\gamma}$ is defined in a similar manner to that for $I$ with $\beta$ replaced by $\gamma$, the function

$$
V^{*}(x)=\frac{1}{1-\gamma} \mathrm{E}\left[\mathbf{1}_{\left\{x+I_{\gamma} \leqslant x^{*}\right\}} g\left(x+X_{\bar{T}}\right)\right]
$$


is the solution for (15), and so also for (14). In other words,

$$
\tilde{V}^{*}(x)=V^{*}(x)+\mathrm{E}\left[\beta^{\tilde{T}} f\left(x+X_{\tilde{T}}\right)\right]
$$

is the solution for (12), while the optimal stopping time is $\tau^{*}=\inf \left\{n \geqslant 0: x+X_{n} \leqslant x^{*}\right\}$.

\subsection{Power reward functions}

Before discussing the case when rewards are power functions, we note that the requirement of Theorem 1 (respectively Theorem 2) that $G(x)$ has the representation (2) in the discrete-time setting (respectively (7) in the continuous-time setting) on the whole of the real line can be modified to allow for the representation to hold only on the set $D=\{x: G(x)>0\}$, under the restriction that $D$ is a semi-infinite interval. We make this precise in the following proposition.

Proposition 1. Let $D=\{x: G(x)>0\}$, and assume that on this set $G$ has the representation (2) in the discrete-time setting (respectively (7) in the continuous-time setting) for some $g$.

(i) If $D$ has the form $(-\infty, h)$ for some $h$, and if there is an $x^{*}<h$ such that (4) (respectively (8)) holds for all $x \in D$, then the result of Theorem 1(i) (respectively Theorem 2(i)) is still valid.

(ii) If $D$ has the form $(h, \infty)$ for some $h$, and if there is an $x^{*}>h$ such that (6) (respectively (9)) holds for all $x \in D$, then the result of Theorem 1(ii)(respectively Theorem 2(ii)) is still valid.

Proof. We outline the proof for the first case in the discrete-time setting. Most of the argument in the proof for Theorem 1(i) follows, except that we need to show that, under the current assumptions, (a) it is still true that $W(x) \geqslant G^{+}(x)$ and (b) the representation (2) holds at $x+X_{\tau^{*}}$. For (a), the inequality $W(x) \geqslant G^{+}(x)$ is true on $D^{\mathrm{c}}$, the complement of $D$, since $W(x) \geqslant 0 \geqslant G(x)$ for $x \in D^{\mathrm{c}}$. When $x$ lies in $D$, our assumptions imply that $x+I>x^{*}$ if and only if $h>x+I>x^{*}$, and so

$$
\mathrm{E}\left[\mathbf{1}_{\left\{x+I>x^{*}\right\}} g\left(x+X_{T}\right)\right] \leq 0 .
$$

Thus,

$$
W(x) \geqslant(1-\beta)^{-1} \mathrm{E}\left[g\left(x+X_{T}\right)\right]
$$

and the representation (2) leads to $W(x) \geqslant G^{+}(x)$, as in the proof for Theorem 1(i). For (b), it follows from the definition of $\tau^{*}$ that $x+X_{\tau^{*}} \in D$, implying that the representation (2) holds at $x+X_{\tau^{*}}$.

In the following, we consider the case when rewards are power functions in the continuoustime setting so that $\left\{X_{t}\right\}_{t \in \mathbb{R}^{+}}$is a Lévy process starting from the origin.

(a) Assume that $\mathrm{E}\left[X_{t}^{2}\right]<\infty$, and let $G(x)=\left(x^{+}\right)^{2}$. Writing $m_{1}$ and $m_{2}$ for the first two moments of $X_{1}$, respectively, we can check that the function $g(x)=r x^{2}-2 m_{1} x-\left(m_{2}-m_{1}^{2}\right)$ satisfies

$$
x^{2}=\int_{0}^{\infty} \mathrm{e}^{-r t} \mathrm{E}\left[g\left(x+X_{t}\right)\right] \mathrm{d} t .
$$

Thus, with this choice of $g, G$ admits the representation (7) on the set $D=\{x: G(x)>0\}=$ $(0, \infty)$ and so Proposition 1(ii) applies subject to its conditions. Using the identity $X_{T} \stackrel{D}{=} I+\tilde{M}$, 
and by direct calculation of $\mathrm{E}\left[X_{T}\right]$ and $\mathrm{E}\left[X_{T}^{2}\right]$ in terms of the moments of $I$ and $M$, we obtain

$$
r^{-1} \mathrm{E}[g(x+I)]=x^{2}-2 \mathrm{E}[M] x+2 \mathrm{E}[M]^{2}-\mathrm{E}\left[M^{2}\right]=\left(x-\kappa_{1}\right)^{2}-\kappa_{2}=Q(x),
$$

where $\kappa_{1}=\mathrm{E}[M]$ and $\kappa_{2}=\mathrm{E}\left[M^{2}\right]-\mathrm{E}[M]^{2}$. It was shown in [10] that $Q(x)$ has a unique positive root $x^{*}$, such that $Q(x)$ is positive increasing for all $x>x^{*}$, and $Q(x) \leqslant 0$ for all $0<x<x^{*}$, thus proving that the conditions for Proposition 1(ii) are satisfied. It then follows that the solution of the corresponding optimal stopping problem (1) is

$$
V^{*}(x)=r^{-1} \mathrm{E}\left[\mathbf{1}_{\left\{x+M \geqslant x^{*}\right\}} g\left(x+X_{T}\right)\right], \quad \tau^{*}=\inf \left\{t: x+X_{t} \geqslant x^{*}\right\},
$$

which is precisely the solution given in [10].

(b) Let noninteger $v>0$, and assume that $\mathrm{E}\left[\left|X_{t}\right|^{v}\right]<\infty$. Consider $G(x)=\left(x^{+}\right)^{v}$. For this, we first define

$$
f_{v}(u)=\frac{v \mathrm{e}^{-(v+1) \ln (\mathrm{i} u)}}{\Gamma(1-v)}
$$

and $P_{k}(x)=\sum_{i=0}^{k} x^{i} / i$ ! for $k \in \mathbb{Z}^{+}$. Then, for positive $x$, it is true that

$$
\int_{0}^{\infty} f_{v}(u)\left\{P_{[v]}(-\mathrm{i} u x)-\mathrm{e}^{-\mathrm{i} u x}\right\} \mathrm{d}(\mathrm{i} u)=x^{\nu} .
$$

For each $k$, we further define a $k$-degree polynomial $R_{k, u}$ such that

$$
\mathrm{E}\left[R_{k, u}\left(x+X_{T}\right)\right]=P_{k}(-u x) .
$$

Using this polynomial, we define

$$
g_{v}(x)=r \operatorname{Re}\left\{\int_{0}^{\infty} f_{v}(u)\left(R_{[v], \mathrm{i} u}(x)-\frac{\mathrm{e}^{-\mathrm{i} u x}}{\mathrm{E}\left[\mathrm{e}^{\left.-\mathrm{i} u X_{T}\right]}\right.}\right) \mathrm{d}(\mathrm{i} u)\right\},
$$

when the right-hand side is defined. It is now easy to see that

$$
\begin{aligned}
\int_{0}^{\infty} \mathrm{E}\left[g_{v}\left(x+X_{t}\right)\right] \mathrm{d} t & =r^{-1} \mathrm{E}\left[g_{v}\left(x+X_{T}\right)\right] \\
& =\operatorname{Re}\left\{\int_{0}^{\infty} f_{v}(u)\left(P_{[\nu]}(-\mathrm{i} u x)-\mathrm{e}^{-\mathrm{i} u x}\right) \mathrm{d}(\mathrm{i} u)\right\} \\
& =x^{\nu},
\end{aligned}
$$

so that $G$ has the representation (7) in terms of $g$ on $(0, \infty)$. Now

$$
\frac{v \mathrm{e}^{-(v+1) \ln (z)}}{\Gamma(1-v)}\left\{\mathrm{E}\left[R_{[v], z}(x+I)\right]-\frac{\mathrm{e}^{-z x}}{\mathrm{E}\left[\mathrm{e}^{-z M}\right]}\right\}
$$

is analytic for $\operatorname{Re}(z)>0$ and continuous for $\operatorname{Re}(z) \geqslant 0$ (see Theorem 1 of [2]). If

$$
\sup _{\substack{|z|=R \\ \operatorname{Re}(z)>0 \\ \operatorname{Im}(z)>0}}\left|\frac{z^{-[v]} \mathrm{e}^{-z x}}{\mathrm{E}\left[\mathrm{e}^{-z M}\right]}\right|
$$


remains bounded for all $R$, then by Cauchy's rule

$$
\begin{aligned}
r^{-1} \mathrm{E}\left[g_{v}(x+I)\right] & =\operatorname{Re}\left\{\mathrm{E}\left[\int_{0}^{\infty} f_{v}(u)\left(R_{[v], i u}(x+I)-\frac{\mathrm{e}^{-\mathrm{i} u(x+I)}}{\mathrm{E}\left[\mathrm{e}^{\left.-\mathrm{i} u X_{T}\right]}\right.}\right) \mathrm{d}(\mathrm{i} u)\right]\right\} \\
& =\operatorname{Re}\left\{\int_{0}^{\infty} f_{v}(u)\left(\mathrm{E}\left[R_{[v], i u}(x+I)\right]-\frac{\mathrm{e}^{-\mathrm{i} u x}}{\mathrm{E}\left[\mathrm{e}^{-\mathrm{i} u M}\right]}\right) \mathrm{d}(\mathrm{i} u)\right\} \\
& =\int_{0}^{\infty} v \frac{\mathrm{e}^{-(v+1) \ln (u)}}{\Gamma(1-v)}\left\{\mathrm{E}\left[R_{[v], u}(x+I)\right]-\frac{\mathrm{e}^{-u x}}{\mathrm{E}\left[\mathrm{e}^{-u M}\right]}\right\} \mathrm{d} u \\
& =Q_{v}(x) .
\end{aligned}
$$

It was shown in [14] that $Q_{v}(x)$ has a unique positive root $x^{*}$ such that $Q_{v}(x)$ is positive increasing for all $x>x^{*}$, and $Q_{v}(x) \leqslant 0$ for all $0<x<x^{*}$. Thus, by Proposition 1 , the solution of the optimal stopping problem with $G(x)=\left(x^{+}\right)^{v}$ is given by

$$
r^{-1} \mathrm{E}\left[\mathbf{1}_{\left\{x+M \geq x^{*}\right\}} g_{v}\left(x+X_{T}\right)\right],
$$

which is the same as the solution given in [14].

One case, in which (16) is satisfied and $g_{v}$ is well defined, is when the characteristic function of the supremum is of the order $(a+|z|)^{-k}$ for positive integer $k$, and $v>2 k$. For example, when $X_{t}$ is Brownian motion or any spectrally negative Lévy process that is not a subordinator, the supremum is exponentially distributed with some parameter $\lambda>0$. Another situation is when the positive jumps of the process have a phase-type distribution. In this case, it has been shown (see [12]) that the supremum also has a phase-type distribution. Phase-type distributions have rational transforms and, if the process is not a subordinator, then its characteristic function is of the form $P(z) / Q(z)$, where the degree of the polynomial $Q$ is one higher than that of the polynomial $P(z)$ (see [1]).

\section{Acknowledgements}

We are grateful to the anonymous referee for the careful reading of this paper and helpful comments. We are also grateful to Professor A. E. Kyprianou for bringing to our attention, after the submission of this paper, the work of Surya [16], who studied the solution to our optimal stopping problem under the Lévy model.

\section{References}

[1] Asmussen, S., Avram, F. And Pistorius, M. R. (2004). Russian and American put options under exponential phase-type Lévy models. Stoch. Process. Appl. 109, 79-111.

[2] Bingham, N. H. (1975). Fluctuation theory in continuous time. Adv. Appl. Prob. 7, 705-766.

[3] Boyarchenko, S. and LevendorskiĬ, S. Z. (2006). General option exercise rules, with applications to embedded options and monopolistic expansion. Contrib. Theoret. Econom. 6, 53 pp.

[4] Boyarchenko, S. and Levendorskĭ, S. (2007). Practical guide to real options in discrete time. Internat. Econom. Rev. 48, 311-342.

[5] Darling, D. A., Liggett, T. and Taylor, H. M. (1972). Optimal stopping for partial sums. Ann. Math. Statist. 43, 1363-1368.

[6] Feller, W. (1971). An Introduction to Probability Theory and Its Applications, Vol. II, 2nd edn. John Wiley, New York.

[7] Greenwood, P. and Pitman, J. (1980). Fluctuation identities for random walk by path decomposition at the maximum. Adv. Appl. Prob. 12, 291-293.

[8] Greenwood, P. and Pitman, J. (1980). Fluctuation identities for Lévy processes and splitting at the maximum. Adv. Appl. Prob. 12, 893-902. 
[9] Kyprianou, A. E. (2006). Introductory Lectures on Fluctuations of Lévy Processes with Applications. Springer, Berlin.

[10] Kyprianou, A. E. And Surya, B. A. (2005). On the Novikov-Shiryaev optimal stopping problems in continuous time. Electron. Commun. Prob. 10, 146-154.

[11] Mordecki, E. (2002). Optimal stopping and perpetual options for Lévy processes. Finance Stoch. 6, $473-493$.

[12] MordecKI, E. (2002). The distribution of the maximum of a Lévy process with positive jumps of phase-type. Theory Stoch. Process. 8, 309-316.

[13] Novikov, A. A. And Shiryaev, A. N. (2005). On an effective case of the solution of the optimal stopping problem for random walks. Theory Prob. Appl. 49, 344-354.

[14] Novikov, A. And Shiryaev, A. (2007). On a solution of the optimal stopping problem for processes with independent increments. Stochastics 79, 393-406.

[15] Rogers, L. C. G. And Williams, D. (2000). Diffusions, Markov Processes, and Martingales, Vol. 1. Cambridge University Press.

[16] Surya, B. A. (2007). An approach for solving perpetual optimal stopping problems driven by Lévy processes. Stochastics 79, 337-361. 